\title{
CD 148, a membrane protein tyrosine phosphatase, is able to induce tyrosine phosphorylation on human lymphocytes
}

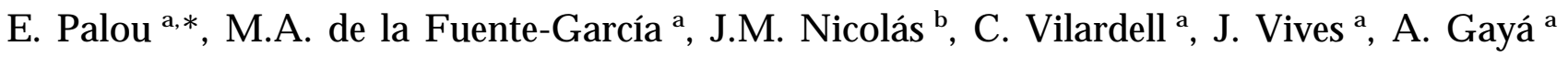 \\ a Servei d'Immunologia, H ospital Clinic Villarroel 170, 08036 Barcelona, Spain \\ b Servei de M edicina Interna, H ospital Clínic, Villarroel 170, 08036 Barcelona, Spain
}

\begin{abstract}
A bstract
CD 148 is a new cluster of differentiation defined in the VI International Workshop on L eucocyte Differentiation A ntigens. It has been identified as the hematopoietic form of a formerly described membrane protein tyrosine phosphatase called HPTP $\eta /$ DEP-1. Previous data have demonstrated that this molecule is able to give rise to $\left[\mathrm{Ca}^{2+}\right]_{i}$ increase. In the present work we show its capability to induce protein tyrosine phosphorylation in human lymphocytes in spite of its intrinsic protein tyrosine phosphatase activity. The induction of kinase activity suggests the involvement of some protein tyrosine kinase based signaling pathway. The activation of this postulated kinase could be carried out through a direct association or via an adapter molecule. (C) 1997 Elsevier Science B.V.
\end{abstract}

Keywords: Protein kinases/phosphatases; Signal transduction; Cell surface molecules; T-lymphocytes

\section{Introduction}

Tyrosine phosphorylation is an essential regulatory mechanism used in signal transduction cascades to control cell proliferation, differentiation and migration $[1,2]$. R egulation of tyrosine phosphorylation is carried out by the concerted activities of protein tyrosine kinases and protein tyrosine phosphatases. Leukocytes express a wide variety of protein tyrosine phosphatases which include cytosolic phosphatases, such as PTP-1C and PTP-1D, or transmembrane phosphatases. A $n$ increasing number of receptor function protein tyrosine phosphatases have been identified [3], CD 45 being the most relevant one.

CD148 was defined during the VI International Workshop on L ymphocyte D ifferentiation A ntigens [4] using two monoclonal antibodies: A 3 and 143.41, the last one produced and studied in our laboratory. The CD 148 molecule is present on different subpopulations of peripheral blood cells with different levels of expres-

* Corresponding author. Tel.: + 343 4544920; fax: + 343 4518038; e-mail: epalou@medicina.ub.es sion, showing its highest expression on granulocytes, and being present at intermediate intensity on monocytes and lymphocytes. After immunopurifying and partial microsequencing the protein, CD 148 was identified as the type III transmembrane protein tyrosine phospahatase HPTP $\eta / D E P-1$ (de la F uente et al. submitted for publication), previously cloned by two independent groups $[5,6]$. This molecule was shown to have an extracellular portion composed of eight or ten fibronectin III domains, a transmembrane segment and an intracytoplasmic tail which contains a single protein tyrosine phosphatase domain, in contrast with most receptor protein tyrosine phosphatases which contain a tandem repeat of two cytoplasmic phosphatase domains. The presence of fibronectin III motifs in the extracellular domains suggests its possible involvement in cell adhesion processes. On the other hand, the existence of the phosphatase domain in the intracytoplasmic tail evidences its participation in signal transduction.

Previous results in our laboratory have shown that CD 148 crosslinking was able to induce $\mathrm{Ca}^{2+}$ mobilization (de la F uente et al. submitted for publication). This 
effect could be abolished by the addition of genistein, a known protein tyrosine kinase inhibitor, as well as by co-crosslinking with CD 45, suggesting the involvement of a tyrosine kinase based signaling. In the present paper we have analyzed the ability of CD 148 to induce tyrosine phosphorylation and compared it with the effect of CD 45, a protein tyrosine phosphatase also present on the membrane of lymphocytes.

\section{2. $M$ aterial and methods}

\subsection{Cells}

Blood samples were obtained from healthy adult donors. Peripheral blood mononuclear cells were isolated by centrifugation over $\mathrm{F}$ icoll-H ypaque (Pharmacia LKB, Uppsala, Sweden) density-gradient sedimentation.

\subsection{M onoclonal antibodies}

The following $\mathrm{mA}$ bs were produced in our laboratory and ascribed to their CDs through one of the International Workshops on H uman L eukocyte D ifferentiation Antigens: 143-41 (CD 148, I gG 1), CRIS-7 (CD 3, IgG 2a) and 72-5D 3 (CD 45, IgG 2a).

\subsection{D etection of tyrosine-phosphorylated proteins}

Cells $\left(5 \times 10^{6}\right)$ were incubated with the different monoclonal antibodies $(10 \mu \mathrm{g})$ for $15 \mathrm{~min}$ at $4^{\circ} \mathrm{C}$ followed by the addition of cross-linking rabbit antimouse immunoglobulins $(5 \mu \mathrm{g})$. Incubation was terminated after different periods of time by the addition of $1 \mathrm{ml}$ of ice-cold stop buffer (50 mM Hepes, $150 \mathrm{mM}$ $\mathrm{NaCl}, 100 \mathrm{mM} \mathrm{NaF}, 10 \mathrm{mM} \mathrm{EDTA}, 10 \mathrm{mM}$ $\mathrm{Na}_{4} \mathrm{P}_{2} \mathrm{O}_{7}, 2 \mathrm{mM}$ Sodium Pervanadate, $2 \mathrm{mM} \mathrm{PMSF}$, $10 \mu \mathrm{g} / \mathrm{ml}$ aprotinin, $10 \mu \mathrm{g} / \mathrm{ml}$ pepstatin, $1 \mu \mathrm{g} / \mathrm{ml}$ leupeptin, $100 \mu \mathrm{M}$ PAO). Cells were pelleted and lysed with stop buffer containing 1\% NP-40. Proteins were separated by SDS-PAGE and transferred to nitrocellulose membranes. Phosphotyrosine-containing proteins were probed with anti-phosphotyrosine monoclonal antibody PY-20 from Santa Cruz Biotechnology (Santa Cruz, CA) and horseradish peroxidase conjugated rabbit anti-mouse and visualized by fluorography with enhanced chemiluminiscence $(E C L)$ reagent (A mersham, Buckinghamshire, UK).

\section{R esults and discussion}

In order to confirm the capacity of CD 148 to induce protein phosphorylation an assay consisting in a phosphotyrosine immunoblot of differently stimulated cell lysates was developed. As can be observed in Fig. 1, after CD 3 crosslinking a neat pattern of protein tyrosine phosphorylation was detected, as has been previously described [7]. In a similar way, after CD 148 crosslinking also a clear pattern of phosphorylation could be observed. Likewise, an increase in tyrosine phosphorylation was observed after crosslinking CD 45, a known membrane protein tyrosine phosphatase used as a control. In addition to the phosphorylation of some substrates in common with both CD 3 and CD 45, CD 148 was able to induce tyrosine phosphorylation of some specific substrates (e.g. $52 \mathrm{Kd}$ ). On the other hand, some substrates clearly hyperphosphorylated after CD 3 (e.g. $56 \mathrm{Kd}$ ) and CD 45 (e.g. 32 $\mathrm{K} d)$ stimulation were not detected after CD 148 stimulation.

These results show that the CD 148 molecule participates in some signal transduction pathway that involves protein tyrosine phosphorylation even though it has a phosphatase activity. This apparent contradiction could be explained by the possible dephosphorylation of some substrates whose kinase activity would be activated by this dephosphorylation. A ctually, the requirement of CD 45 in antigen-induced receptor signaling is based on its regulatory capacity on the Srcfamily protein tyrosine kinases p56 lck and p59fyn [8]. By dephosphorylating a negative regulatory tyrosine

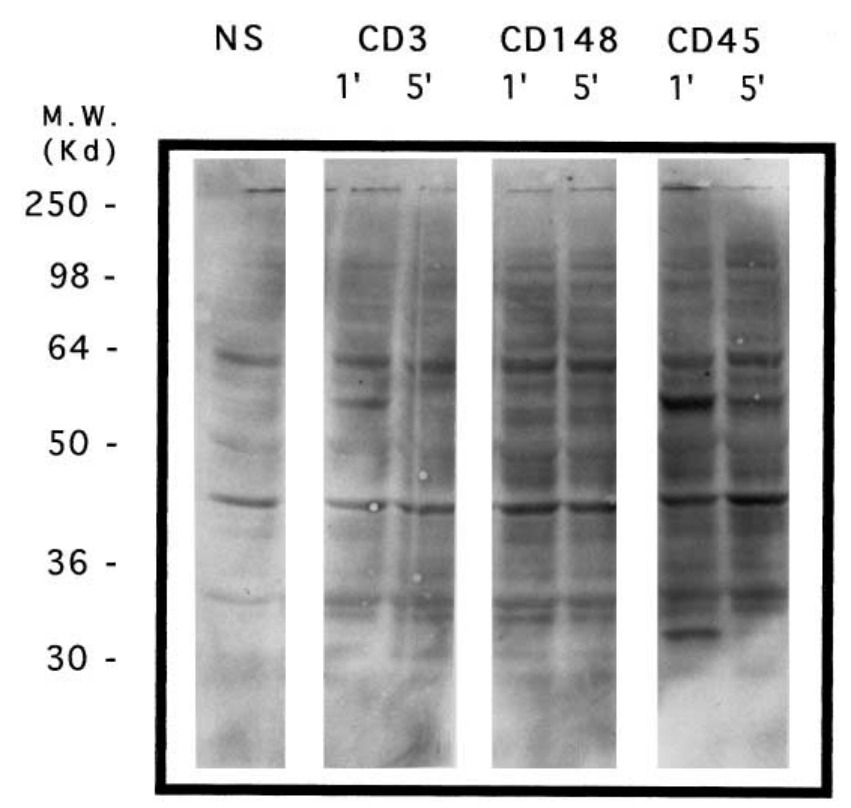

Fig. 1. CD 148 crosslinking induces tyrosine phosphorylation on lymphocytes. Cells were left untreated (NS) or incubated with CD 3, CD 148 or CD 45 and after washing, goat anti-mouse was added. The cells were incubated during the indicated times, the reaction was stopped by the addition of stop buffer and cells were lysed with lysis buffer. Total lysates were subjected SDS-PAGE, transferred to nitrocellulose paper and tyrosine phosphorylated proteins were detected by immunoblot with anti-phosphotyrosine antibody. 
phosphorylation site at their carboxyl termini, CD 45 causes an increase in kinase activity. In fact, it has been shown that p56 ck co-immunoprecipitates with CD 45 thus establishing a link between these two molecules [9]. Although we have no data on the effect of CD 148 on p56 lck, no clear phosphorylation of substrates around $56 \mathrm{Kd}$ was detected after CD 148 stimulation. In any case, the observed increase in tyrosine phosphorylation suggests the implication of some tyrosine kinase which could interact with CD 148 directly or through an adapter.

The 3BP2 protein is a possible candidate as an adapter molecule. A potential binding site of the $\mathrm{SH} 2$ domain of the $3 B P 2$ protein, a YEN (L/V) motif, has been described by $\mathrm{K}$ uramochi et al. [10] in the cytoplasmic tail of byp, the murine equivalent of human CD 148. The presence of this motif is also observed in the cytoplasmic tail of human CD 148. The 3BP2 protein contains a proline-rich domain and a plekstrin-homology domain (PH). The PH domain has been found on several intracellular proteins implicated in signaling [11] and has been postulated to mediate regulated protein-protein interactions during signaling, in a similar manner to the SH 2 domain. In addition to CD 148 and other type III human phosphatases like HPTP- $\beta$ and GLEPP-1, the 3BP2 binding domain is found on some other proteins present in the membrane of haematopoietic cells: CD 19, $C D 72$, human high affinity epsilon receptor, human EPO receptor and human G-CSFR [12]. A common characteristic of all these molecules is their capability to induce tyrosine phosphorylation. Whether the 3BP2 molecule is implicated on the signal transduction pathway through CD148 or there is a direct interaction with some protein tyrosine kinase remains to be elucidated.

\section{R eferences}

[1] M. Streuli, Protein tyrosine phosphatases in signaling, Curr. Opin. Cell Biol. 8 (1996) 182-188.

[2] J.A. F rearson, D.R. A lexander, Protein tyrosine phosphatases in T-cell development, apoptosis and signalling, Immunol. Today 17 (1996) 385- 391.

[3] M. Okumura, M .L. Thomas, R egulation of immune function by protein tyrosine phosphatases, Curr. Opin. Immunol. 7 (1995) $312-319$.

[4] B. Schraven, M. H egen, F. A utschbatch, A. G ayá, C. Schwartz, S. M euer, in: K ishimoto, (Ed.), Cluster R eport CD 148-L eucocyte Typing VI, Garland Publishing, N ew Y ork, 1997, In press.

[5] H. H onda, J. Inazawa, J. N ishida, Y. Y azaki, H. H irai, M olecular cloning, characterization, and chromosomal localization of a novel protein-tyrosine phosphatase, HPTP $\eta$, Blood 84 (1994) 4186- 4194.

[6] A. Östman, Q. Y ang, N.K. Tonks, Expression of DEP-1, a receptor-like protein-tyrosine-phosphatase, is enhanced with increasing cell density, Proc. N atl. A cad. Sci. U SA 91 (1994) 9680- 9684.

[7] G. Zenner, J.D. H ausen, P. Burn, T. M ustelin, Towards unraveling the complexity of T-cell signal transduction, Bioessays 17 (1995) 967-975.

[8] J.A. L ebdetter, N.K. Tonks, E.H. Fisher, E.A. Clark, CD 45 regulates signal transduction and lymphocyte activation by specific association with receptor molecules on $\mathrm{T}$ or $\mathrm{B}$ cells, Proc. N atl. A cad. Sci. U SA 85 (1988) 8628-8632.

[9] D.H.W. N g, J.D. W atts, R. A ebersold, P. Johnson, D emonstration of a direct interaction between p56 lck and the cytoplasmic domain of CD 45 in vitro, J. Biol. Chem. 271 (1996) 1295-1300.

[10] S. K uramochi, S. M atsuda, Y. M atsuda, T. Saitoh, M. Ohsugi, T. Y amamoto, M olecular cloning and characterization of Byp, a murine receptor-type tyrosine phosphatase similar to human DEP-1, FEBS L etters 378 (1996) 7-14.

[11] B.J. Mayer, R. Ren, K.L. Clark, D. Baltimore, A putative modular domain present in diverse signaling proteins, Cell 73 (1992) 629-630.

[12] Z. Songyang, S.E. Shoelson, J. M cG lade, P. Oliver, T. Pawson, $X . R$. Bustelo, M. Barbacid, H. Sabe, H. H anafusa, T. Y i, R. Ren, D. Baltimore, S. R atnofsky, R.A. F eldman, L.C. Cantley, Specific motifs recognized by the $\mathrm{SH} 2$ domains of $\mathrm{Csk}, 3 \mathrm{BP} 2$, $\mathrm{fps} / \mathrm{fes}, \mathrm{GRB}-2, \mathrm{HCP}, \mathrm{SHC}$, Syk, and Mol. Cell Biol. 14 (1994) 2777-2785. 